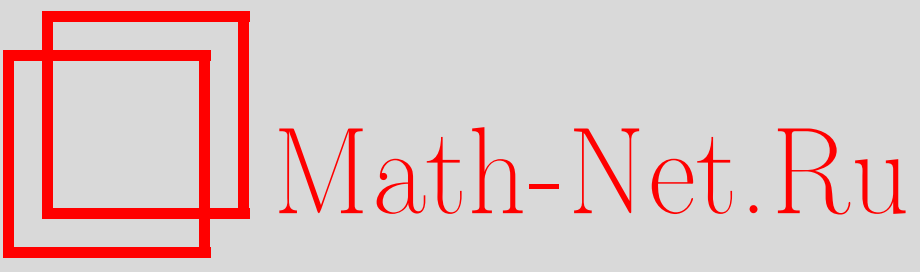

В. В. Балащенко, Естественно редуктивные киллинговы $f$-многообразия, $У M H$, 1999, том 54, выпуск 3, 151-152

DOI: https://doi.org/10.4213/rm155

Использование Общероссийского математического портала Math-Net.Ru подразумевает, что вы прочитали и согласны с пользовательским соглашением

http://www.mathnet.ru/rus/agreement

Параметры загрузки:

IP : 54.172 .240 .79

26 апреля 2023 г., 16:30:55 


\title{
ЕСТЕСТВЕННО РЕДУКТИВНЫЕ КИЛЛИНГОВЫ $f$-МНОГООБРАЗИЯ
}

\author{
В. В. БАЛАШЕНКО
}

Среди почти эрмитовых структур $(g, J)$ на гладких многообразиях $M$ одним из самых примечательных классов являются приближенно келеровы структуры (см., например, [1]). Определяющим условием для них является требование $\nabla_{X}(J) X=0$, где $\nabla$ - связность Леви-Чивита (псевдо-) римановой метрики $g, X$ - произвольное гладкое векторное поле на $M$. Обобщением этого понятия на метрические $f$-структуры [2] классического типа $\left(f^{3}+f=0\right)$ стали киллинговы $f$-структуры, определяемые условием $\nabla_{X}(f) X=0$. Впервые такие структуры под другим названием были введены В.Ф. Кириченко в работе [3], а затем исследовались А.С. Грицансом (см. [4], [5]). Позднее автором заметки (см. [6]) был рассмотрен более общий класс - приближенно келеровы $f$-структуры (nearly Kähler $f$-structures): $\nabla_{f X}(f) f X=0$. Заметим, что оба указанных обобщения входят в концепцию обобщенной эрмитовой геометрии и принадлежат широкому классу обобщенных $G_{1}$-многообразий (см. [2]).

Замечательной особенностью в исследованиях приближенно келеровых структур стало наличие широкого класса инвариантных примеров, что позволило получить ряд глубоких геометрических результатов (см. [7]-[9]). Природа этих примеров связана с каноническими структурами на обобщенных симметрических пространствах. Оказалось [6], что эта связь допускает содержательное продолжение и позволяет предъявить большой класс инвариантных примеров приближенно келеровых $f$-структур. Что касается киллинговых $f$-структур, исследование которых интенсивно продолжается (см., например, [10]), то здесь нетривиальных инвариантных примеров до сих пор обнаружено не было.

В настоящей заметке анализируются возможности существования инвариантных киллинговых $f$-структур на естественно редуктивных однородных пространствах. В частности, получен ряд критериев киллинговости для канонических $f$-структур на однородных $\Phi$-пространствах порядков 4 и 5 .

Пусть $(G / H, g, f)$ - однородное пространство группы Ли $G$ с инвариантной метрикой $g$ и инвариантной метрической $f$-структурой. Для простоты будем обозначать одинаковыми символами как сами инвариантные структуры на $G / H$, так и их значения в точке $o=H$. Предположим, что $G / H$ - редуктивное однородное пространство, $\mathfrak{g}=\mathfrak{h} \oplus \mathfrak{m}-$ соответствующее редуктивное разложение алгебры Ли $\mathfrak{g}$ группы $G$. Напомним, что $(G / H, g)$ называется естественно редуктивныцм пространством, если $g\left([X, Y]_{\mathfrak{m}}, Z\right)=g\left(X,[Y, Z]_{\mathfrak{m}}\right)$ для всех $X, Y, Z \in \mathfrak{m}$, где индекс $\mathfrak{m}$ обозначает проекцию на $\mathfrak{m}$ относительно редуктивного разложения. Задание на $G / H$ инвариантной метрической $f$-структуры порождает ортогональное разложение $\mathfrak{m}=\mathfrak{m}_{1} \oplus \mathfrak{m}_{2}$, где подпространства $\mathfrak{m}_{1}$ и $\mathfrak{m}_{2}$ определяют соответственно первое и второе фундаментальные распределения для $f$.

Теорема 1. Пусть $(G / H, g, f)$ - естественно редуктивное киллингово $f$-многообразие. Тогда имеют место соотношения:

$$
\left[\mathfrak{m}_{1}, \mathfrak{m}_{1}\right] \subset \mathfrak{m}_{1} \oplus \mathfrak{h}, \quad\left[\mathfrak{m}_{2}, \mathfrak{m}_{2}\right] \subset \mathfrak{m}_{2} \oplus \mathfrak{h}, \quad\left[\mathfrak{m}_{1}, \mathfrak{m}_{2}\right] \subset \mathfrak{h}
$$

В частности, оба фундаментальных распределения киллинговой $f$-структуры определяют инвариантные вполне геодезические слоения многообразия $G / H$.

С учетом результатов работы [4] отсюда получаем

СлЕДСтвИЕ. На естественно редуктивном однородном пространстве $(G / H, g)$ не существует нетривиальных инвариантных киллинговых $f$-структур основного тиna [4].

Отмеченный факт широко обобщает соответствующий результат А. С. Грицанса, полученный для римановых глобально симметрических пространств.

Теорема 2. Для естественно редуктивного киллингова $f$-многообразия $(G / H, g, f)$ тензор Нейенхейса $N$ и композиционный тензор $T[2]$ имеют вид: $N(X, Y)=$ $[f X, f Y]_{\mathfrak{m}}=2 T(X, Y)$, əде $X, Y \in \mathfrak{m}$. В частности, критерием интегрируемости структуры $f$ является условие $\left[\mathfrak{m}_{1}, \mathfrak{m}_{1}\right] \subset \mathfrak{h}$.

Перейдем теперь к рассмотрению регулярных $\Phi$-пространств, на которых имеется значительньй запас канонических $f$-структур [11]. 
Пусть $G / H$ - регулярное $\Phi$-пространство, порождаемое автоморфизмом $\Phi$ связной группы Ли $G, \mathfrak{g}=\mathfrak{h} \oplus \mathfrak{m}$ - каноническое редуктивное разложение, соответствующее автоморфизму $\varphi=d \Phi_{e}$ алгебры Ли $\mathfrak{g}$, т.е. $\mathfrak{m}=A_{\varphi} \mathfrak{g}, A_{\varphi}=\varphi$-id [12]. Обозначим через $\theta$ ограничение $\varphi$ на $\mathfrak{m}$. Напомним, что инвариантная аффинорная структура $F$ на $G / H$ называется канонической [11], если ее значение в точке $o$ есть полином от $\theta: F=F[\theta]$. В работе [11] получены, в частности, формулы для всех канонических структур типов $J$ и $f$ на однородных $\Phi$-пространствах произвольного порядка $n\left(\Phi^{n}=\mathrm{id}\right)$, которые всегда регулярны (см. [12]). Например, на однородных $\Phi$-пространствах порядка 3 имеется широко известная каноническая структура $J=\frac{1}{\sqrt{3}}\left(\theta-\theta^{2}\right)$, обеспечивающая основной класс примеров инвариантных приближенно келеровых структур (см. [8], [9]). Напомним также (см. [12]), что в случае полупростой группы Ли $G$ любое регулярное $\Phi$-пространство $(G / H, g)$ является естественно редуктивным, где метрика $g$ порождена формой Киллинга алгебры Ли $\mathfrak{g}$.

Перейдем теперь к рассмотрению канонических $f$-структур на однородных $\Phi$-пространствах порядков 4 и 5.

Теорема 3. Пусть $(G / H, g, f)$ - естественно редуктивное Ф-пространство порядка 4 , где $f=\frac{1}{2}\left(\theta-\theta^{3}\right)-$ каноническая метрическая $f$-структура. Структура $f$ киллингова тогда и только тогда, когда $G / H$ - локально симметрическое пространство $($ m.e. $[\mathfrak{m}, \mathfrak{m}] \subset \mathfrak{h})$, для которого $\left[\mathfrak{m}_{1}, \mathfrak{m}_{2}\right]=0$.

Пусть теперь $G / H$ - однородное $\Phi$-пространство порядка 5. Формулы для всех классических канонических структур на $G / H$ получены в [11]. Не приводя их здесь, укажем строение интересующих нас канонических структур. Пусть $\mathfrak{m}=\mathfrak{m}_{1} \oplus \mathfrak{m}_{2}$ - разложение, определяемое канонической структурой почти произведения $P$. Тогда в терминах этого разложения канонические почти комплексные структуры и $f$-структуры имеют вид: $J_{1}=(I, J), J_{2}=(I,-J), f_{1}=(I, 0)$, $f_{2}=(0, J)$, где $I$ и $J$ - специальные операторы комплексных структур на $\mathfrak{m}_{1}$ и $\mathfrak{m}_{2}$ соответственно.

ТеОрема 4. Пусть $(G / H, g)$ - естественно редуктивное Ф-пространство порядка 5 , $f_{1}$ и $f_{2}, J_{1}$ и $J_{2}$ - канонические структуры на этом пространстве. Следующие условия эквивалентны: 1) $f_{1}$ - киллингова структура; 2) $f_{2}$ - киллингова структура; 3) $J_{1}$ и $J_{2}$ являются приближенно келеровыми структурами; 4) $\left[\mathfrak{m}_{1}, \mathfrak{m}_{2}\right]=0$; 5) $G / H$ - локально симметрическое пространство: $[\mathfrak{m}, \mathfrak{m}] \subset \mathfrak{h}$.

ЗАмЕчАниЕ 1. Эквивалентность двух последних условий теоремы 4 можно получить, детализируя общие результаты работы [13].

ЗАмечАниЕ 2. Канонические $f$-структуры на естественно редуктивных $\Phi$-пространствах порядков 4 и 5 могут быть киллинговыми лишь на келеровых многообразиях $G / H$. Это свидетельствует о жесткости условия киллинговости для инвариантных $f$-структур.

ЗАМЕчАниЕ 3 . Вопрос о существовании инвариантных киллинговых $f$-структур основного типа остается открытым.

\section{СПИСОК ЛИТЕРАТУРЫ}

[1] Gray A. // J. Differential Geom. 1970. V. 4. № 3. Р. 283-309. [2] Кириченко В. Ф. // Итоги науки и техн. Пробл. геом. Т. 18. М.: ВИНИТИ, 1986. С. 25-71. [3] Кириченко В.Ф. // Дифференциальная геометрия. Саратов, 1981. С. 43-49. [4] Грицанс А. С. // УМН. 1990. Т. 45. № 4. С. 149-150. [5] Грицанс А. С. // Изв. вузов. Матем. 1992. № 6. С. 49-57. [6] Balashchenko V. V. Riemannian geometry of canonical structures on regular $\Phi$-spaces // Preprint № 174/1994. Ruhr-Universität Bochum, 1994. P. 1-19. [7] Wolf J., Gray A. // J. Differential Geom. 1968. V. 2. № 1-2. P. 77-159. [8] Gray A. // J. Differential Geom. 1972. V. 7. № 3-4. Р. 343-369. [9] Кириченко В.Ф. // Матем. заметки. 1981. Т. 30. № 4. С. 569-582. [10] Кириченко В.Ф., Липагина Л. В. Постоянство типа коммутативно-киллинговых $f$-многообразий. М.: МПГУ, 1998; Деп. в ВИНИТИ РАН. №1564-В98. [11] Балащенко В. В., Степанов Н. А. // Матем. сб. 1995. Т. 186. № 11. С. 3-34. [12] Степанов Н. А. // Изв. вузов. Матем. 1967. № 3. С. 88-95. [13] Чурбанов Ю. Д. // Изв. вузов. Матем. 1994. № 2. С. 84-86. 